\title{
El Adulto Mayor. Un nuevo reto en El Milenio
}

Use of physical exercise for prophylactic purposes in the elderly

MSc. Eugenio Rodolfo González Pérez. ${ }^{1} \&$ DrC Carlos Manuel Fuentes. ${ }^{2}$

Recibido: 08-04-2018 / Revisado: 09-05-2018 / Aceptado: 10-06-2018 / Publicado: 04-07-2018

\section{DOI: https://doi.org/10.33262/anatomiadigital.v1i2.1043}

\begin{abstract}
.
A review of depression in the elderly was carried out with the aim of exposing important aspects related to diagnosis, evolution, prognosis and treatment in order to contribute to a wider understanding of this mental disorder in the elderly population, taking into account the constant growth of this population and presenting depression as an entity of relevant importance because it is one of the most frequent mental disorders at this age. An updated overview was also presented on some components of the health status of the Cuban population aged 60 and over based on statistical data provided by official statistical information systems. An increase in population ageing was observed between 1970 and 2003, with $14.5 \%$ of the population over 60 years of age in 2003. Care for the elderly has been consolidated by
\end{abstract}

\section{Resumen.}

Se realizó una revisión de la depresión en el adulto mayor con el objetivo de exponer aspectos importantes relacionados con el diagnóstico, evolución, pronóstico y tratamiento para contribuir a una comprensión más amplia de este desorden mental en la población anciana, teniendo en cuenta el constante crecimiento de esta población y presentándose la depresión como una entidad de relevante importancia por ser uno de los más frecuentes trastornos mentales a esta edad. También se presentó un panorama actualizado sobre algunos componentes del estado de salud de la población cubana de 60 años de edad y más basado en los datos estadísticos suministrados por los sistemas de información estadísticos oficiales. $\mathrm{Se}$ observó un incremento del envejecimiento

1 Facultad de Ciencias Médicas de Mayabeque, Departamento Cultura Física, Mayabeque, Cuba, ergonzales@infomed.sld.cu iD https://orcid.org/0000-0002-7353-0172

2 Facultad de Ciencias Médicas de Mayabeque, Mayabeque, Cuba, cfuentes@infomed.sld.cu https://orcid.org/0000-0002-7353-0173 
increasing the number of geriatricians as well as the units providing health services to this sector of the population. The main causes of death correspond to chronic noncommunicable diseases, the first five of which are: heart disease, malignant tumors, cerebrovascular disease, influenza and pneumonia, and diseases of the arteries, arterioles and capillaries. In these ages the greatest risks of illness corresponded to acute respiratory infections and acute diarrheal diseases. High blood pressure was the most prevalent disease affecting women. The ageing of the Cuban population is one of the most important demographic and social implications of the decline in fertility, mortality and increased life expectancy.

Keywords: Older adults, aging, disease. poblacional entre 1970 y el 2003, para este último año el 14,5\% de la población rebasaba los 60 años de edad. La atención al adulto mayor se ha ido consolidando a partir del aumento del número de geriatras así como las unidades prestadoras de servicios de salud a este sector de la población. Las principales causas de muerte corresponden a las enfermedades crónicas no transmisibles siendo las 5 primeras: enfermedades del corazón, tumores malignos, enfermedades cerebro vasculares, influenza y neumonía y enfermedades de las arterias, arteriolas y vasos capilares. En estas edades los mayores riesgos de enfermar correspondieron a las infecciones respiratorias agudas y a las enfermedades diarreicas agudas. La hipertensión arterial constituyó la enfermedad de más alta prevalencia con mayores afectaciones para el sexo femenino. El envejecimiento de la población cubana es una de las implicaciones demográficas y sociales más importantes derivadas del descenso de la fecundidad, la mortalidad y el aumento de la esperanza de vida.

Palabras claves: Adultos mayores, envejecimiento, enfermedades

\section{Introducción.}

La longevidad de los ancianos aumenta en todo el mundo paralelamente a la esperanza de vida. La expectativa para las mujeres como promedio es de 67 años y para los hombres de 63 años. Un factor que ha influido de forma relevante en el aumento de la expectativa de vida y su calidad en la población es la actividad física sistemática y bien dirigida, educando y desarrollando así la voluntad y las capacidades físicas; donde ejercen un importante papel en la personalidad y el mejoramiento de su organismo. A medida que las personas envejecen se producen evidencias de modificaciones y alteraciones en su estado de salud física y sicológica. Estos cambios son progresivos e inevitables pero se ha demostrado en varias investigaciones, que el ritmo de degeneración se puede modificar con la actividad física. En efecto, el ejercicio puede ayudar a mantener o mejorar la condición física, el estado mental y los niveles de presión arterial de los ancianos. La Educación Física y los deportes ofrecen grandes posibilidades de actividades a realizar con las personas adultas mayores por lo que 
no podemos encasillar a este grupo atareo específicamente en una forma organizativa o nombre, cualquier forma de participación es válida, siempre y cuando se permita cumplir debidamente los objetivos, lo fundamental es que la actividad que se proponga este adecuadamente adaptadas a las posibilidades del grupo, teniendo en cuenta la adecuación de los mismos y el lugar oportuno donde se enmarquen dentro del programa o plan que se establezca, así como el trabajo didáctico que permitan que todos los participantes lo realicen con éxito.

\section{Desarrollo}

El Envejecimiento: En la edad adulta se comienza a sufrir el deterioro orgánico de la evolución. En este momento de vida se detiene la madurez de las funciones orgánicas, pero a partir de ahí comienza un período involuntario del que nadie puede escapar. Es un fenómeno irreversible que incluye cambios estructurales y funcionales. Es común a todas las especies, aunque depende también de las condiciones socioeconómicas en las que se ha desarrollado el individuo como ser social. Provoca una disminución de la capacidad de adaptación. El aumento de las probabilidades de muerte según pasa el tiempo, como consecuencia final del proceso. ${ }^{(1)}$.

\section{Clasificación del envejecimiento según la OPS:}

- Tercera Edad

- Adulto Mayor

- Cuarta Edad
60 años

más de 65 años

80 años en adelante

\section{Consecuencias sociales del envejecimiento:}

- Aislamiento familiar

- Marginación en la comunidad

- Dependencia

- Carga social

- Melancolía

- Muerte

\section{Efectos del envejecimiento en el sistema nervioso:}

- Se alteran la atención y la concentración

- Disminución de la capacidad sensorial (auditiva, visual)

- Enlentecimiento mental

- Disminución de reflejos

- Menor coordinación y equilibrio

- Disminuye la velocidad de las reacciones y aumenta su período latente.

- Lentitud en la formación y consolidación de los reflejos condicionados y sus relaciones.

- Disminución de la capacidad de reacción compleja. 
- Prolongación del período latente de las reacciones del lenguaje.

- Irritabilidad, dispersión de la atención e inestabilidad emocional.

- Se dificultan los movimientos que requieren una elevada capacidad de coordinación y precisión.

\section{Efectos del envejecimiento sobre el sistema músculo-esquelético}

- Disminución de tejido muscular y óseo

- Disminución de la fuerza, rapidez y coordinación

- Disminución de la movilidad articular

- Se extiende el tiempo de recuperación del organismo

- Disminución del tono muscular.

- Tendencia a la fatiga muscular.

- Los huesos se hacen más frágiles

- La aparición de Osteoporosis y Osteomalacia. .

- Alteraciones progresivas de la columna vertebral.

- Artrosis (asociado a fracturas esguinces, micro traumatismos repetidos)

Efectos del envejecimiento sobre el aparato circulatorio:

- Disminuye el gasto cardiaco

- Disminuye el tono venoso

- Aumenta la rapidez arterial

- Aumenta la presión arterial

\section{Efectos del envejecimiento sobre el aparato respiratorio:}

- Aumenta el volumen residual

- Disminuye la capacidad vital

- Disminuyen los volúmenes funcionales

- Disminuye la ventilación voluntaria máxima.

\section{Cambios fisiológicos del envejecimiento:}

A medida que las personas envejecen, se producen modificaciones en su estado de salud: se alteran las estructuras y se reducen las funciones de las células y los tejidos de todos los sistemas del organismo. Aparecen cambios que afectan:

- La masa metabólica activa

- El tamaño y función de los músculos

- El sistema esquelético

- El aparato cardiovascular

- Los riñones

- Las glándulas sexuales 
- Los receptores sensoriales

- La médula ósea y los glóbulos rojos.

Estos cambios son progresivos e inevitables, pero se ha demostrado con varias investigaciones que el ritmo de algunos se puede modificar con la actividad física.

El adulto mayor es el término o nombre que reciben quienes pertenecen al grupo etáreo que comprende personas que tienen más de 65 años, por lo general, se considera que los adultos mayores, sólo por haber alcanzado este rango de edad, son lo que se conocen como pertenecientes a la tercera edad, o ancianos. Este pasa por una etapa de la vida que se considera como la última, en la que los proyectos de vida ya se han consumado, siendo posible poder disfrutar de lo que queda de vida con mayor tranquilidad. ${ }^{(2)}$

A medida que las personas envejecen se producen evidencias de modificaciones y alteraciones en su estado de salud física y sicológica. Estos cambios son progresivos e inevitables, pero se ha demostrado en varias investigaciones, que el ritmo de degeneración se puede modificar con la actividad física. En efecto, el ejercicio puede ayudar a mantener o mejorar la condición física, el estado mental y los niveles de presión arterial de los ancianos. (3).

\section{Beneficios del Ejercicio para las Personas Mayores. Las Enfermedades:}

Con ejercicios aeróbicos adecuados, aunque estos se empiezan a la edad de 60 años, se puede incrementar de uno a dos años la esperanza de vida, la independencia funcional, y ayudar a prevenir enfermedades. Se ha encontrado que las dos terceras parte de las personas con más de 60 años practican de manera irregular alguna actividad física o son totalmente sedentarios. Por causa de la inactividad aparecen los riesgos de enfermedades crónicas como las cardiovasculares, la hipertensión, la diabetes, la osteoporosis y la depresión. Muchas investigaciones han demostrado que la práctica física regular incrementa la habilidad de un adulto mayor en sus qué áceres diarios, reduce los riesgos de enfermedades crónicas específicas, incluyendo las enfermedades coronarias y baja la tasa de mortalidad. Los sistemas más susceptibles al cambio con el ejercicio son:

- El cardiovascular (modulador de la variabilidad de la frecuencia cardiaca con ejercicio aeróbico)

- El respiratorio

- El inmunológico;

Además, se ven beneficiados:

- La masa metabólica activa

- Los huesos 
- Los músculos

- Los riñones y los receptores sensoriales.

- El ejercicio logra ayudar en el tratamiento y la prevención de la osteoporosis. Se puede mejorar significativamente la calidad de vida de una persona de la tercera edad dándole mayor flexibilidad, fuerza y volumen muscular, movilidad y mayor capacidad funcional aeróbica. ${ }^{(4)}$.

El Estado Mental: El ejercicio logra ayudar en el tratamiento y la prevención de la osteoporosis. También se ha encontrado que el ejercicio es benéfico para la salud mental en la tercera edad. El ejercicio mejora la auto-estima, el autocontrol, el funcionamiento autónomo, los hábitos de sueño, las funciones intelectuales y las relaciones sociales. Cuando se investigaron los estados de ánimos positivos y negativos y la fatiga de mujeres después de una clase de aeróbicos, se encontró una diferencia significativa entre los estados de ánimos antes y después de la clase: el efecto positivo se incrementó y la fatiga se redujo. ${ }^{(5)}$.

La Hipertensión: Los adultos mayores hipertensos que participaron en un programa de ejercicio físico pudieron demostrar que su nivel de presión arterial logró controlarse y hasta se mejoró. En una investigación sobre la presión arterial en reposo de personas mayores con presión arterial normal, los resultados mostraron que los dos grupos de ejercicios, uno de intensidad moderada $(70 \%)$ / 45minutos / 3 veces por semana y el otro de alta intensidad $(85 \%) / 35$ minutos/3 veces por semana aumentaron su consumo máximo de oxígeno trabajando en los tres primeros meses del programa y bajaron su presión arterial sistólica a los seis meses.

\section{Recomendaciones para empezar una Actividad Física:}

En programas de ejercicios supervisados para la tercera edad, los factores de riesgos de lesiones, dolores, accidentes y hasta muerte se encontraron a una intensidad más alta de $85 \%$ de la frecuencia cardiaca máxima; de hecho, el ejercicio excesivamente intenso o prolongado puede conducir a una fatiga extrema, un cansancio y una convalecencia que retarda los beneficios del ejercicio. ${ }^{(6)}$.

Se recomienda una actividad física regular (de 3 hasta 4 veces por semana) de duración moderada (de 20 hasta 30 minutos) y de una intensidad suficiente (de $70 \%$ hasta $85 \%$ ) para obtener efectos de acondicionamiento, minimizando los riesgos de la actividad física. Esta tesis es respaldada por Brown \& Holloszy los cuales investigaron los efectos de la caminata, el trote y la bicicleta sobre la fuerza, la velocidad, la flexibilidad y el equilibrio de personas de 60 hasta 72 años. Tomando en cuenta las diferencias de la población de la tercera edad, el Colegio Americano de Medicina Deportiva recomienda un programa de entrenamiento que sigua los lineamientos básicos de prescripción de la frecuencia, intensidad, duración y tipo 
de actividad para un adulto sano, siendo diferente en su aplicación. Un adulto mayor es diferente y tiene mayores limitaciones física-médicas que un participante de edad mediana, por esto la intensidad del programa es menor mientras la frecuencia y duración se incrementan. El tipo de entrenamiento debe evitar las actividades de alto impacto, además debe permitir progresiones más graduales y mantener una intensidad de un $40 \%$ hasta un $85 \%$ de la frecuencia cardiaca máxima. Es también importante incluir en el programa un entrenamiento de mantenimiento de la tonicidad y resistencia de los grandes grupos de musculares. ${ }^{(7)}$.

Clasificación de los Juegos en la Actividad Física:

Activos: Influyen en el desarrollo del movimiento pero que no provoquen accidentes, no realizar acciones bruscas. Se utilizan en el intermedio o final de la parte principal de la clase.

Pasivos: Se realizan en la parte final de la clase como forma amena de que el organismo se recupere.

Métodos que se utilizan para preparar las clases: Se realiza de forma consecutiva cualquier acción motora para consolidar los hábitos y el mantenimiento de cualquier capacidad física.

De competencia: Para perfeccionar hábitos creados con el objetivo de mantener el desarrollo físico alcanzado (de forma amena y motivacional)

De juego: Para desarrollar las capacidades físicas y mantener la motivación y el interés de los participantes.

\section{Objetivos de las Clases del Adulto Mayor:}

- Reincorporar este grupo a las labores y actividades de la sociedad, logrando el fin de la rehabilitación.

- Este programa va dirigido a aquellos ancianos que radican en sus propios hogares y que en horas de la mañana o en la hora que el grupo decida se trasladan a parques, solares, instalaciones deportivas a recibir clases de ejercitación física por medio de instructores de Cultura física o

- Monitores miembros del propio grupo.

- Ejercer una acción estimulante sobre los procesos del sistema nervioso central.

- Elevar las posibilidades funcionales de los sistemas cardiovascular y respiratorio.

- Mejorar los procesos metabólicos.

- Fortalecer el sistema muscular.

- Incrementar la movilidad articular.

- Mantener el nivel de los hábitos y destreza motoras vitales. ${ }^{(8)}$.

\section{Beneficios de la Práctica del Ejercicio Físico:}


- Sistema Nervioso: La coordinación y los reflejos mejoran, el estrés disminuye.

- Corazón: El volumen de sangre por pulsación y la circulación coronaria

- aumentan.

- Pulmones: La capacidad, eficiencia y circulación aumentan.

- Músculos: La circulación en ellos aumenta, agrandando el tamaño, la fuerza y la resistencia, así como la capacidad de oxigenación.

- Metabolismo: Las grasas del cuerpo disminuyen; el azúcar de la sangre se reduce.

\section{Panorama de Salud del Adulto Mayor en Cuba:}

\section{Características Demográficas:}

- En 1970 la población de 60 años y más (adulto mayor o población de la tercera edad) constituía el 9,0\% del total.

- En el año 2003, esta población asciende a 1630185 lo que representa el 14,5\% del total.

- La población femenina predomina discretamente debido a su mayor expectativa de vida: 51,8 \% las mujeres y 48,2 \% los hombres (fig. 1).

Figura N 1. Porcentaje de población de 60 años y más.

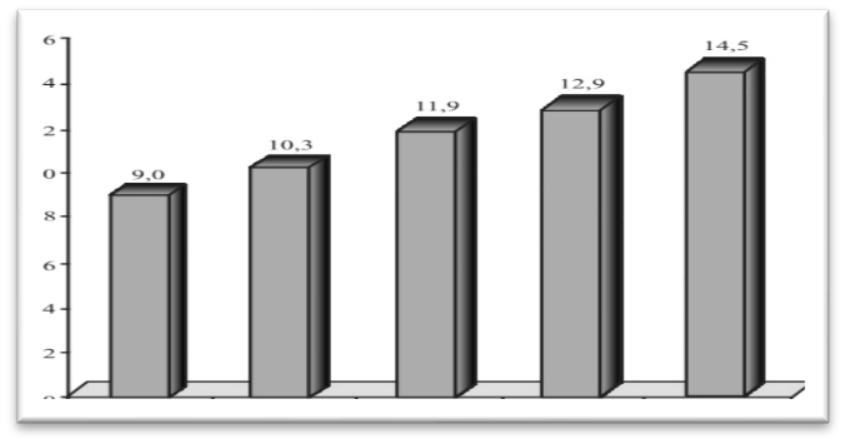

- El 54,6 \% de la población adulta mayor reside en zonas urbanas, el $29 \%$ en zonas intermedias o también llamadas urbano-rurales y el 16,4 \% en zonas rurales.

- Las provincias más envejecidas del país en el 2003 fueron, Villa Clara y Ciudad de La Habana, con un 17,4 \% y 17,1 \% respectivamente de población de 60 años y más con respecto al total.

\section{Programa de Atención Del Adulto Mayor:}

En 1974 surge el Programa de Atención Integral al Adulto Mayor que encaminó sus esfuerzos al desarrollo de la Geriatría. Posteriormente con el surgimiento del plan del médico y la 
enfermera de la familia se integra a éste la atención al adulto mayor, desarrollándose las alternativas de atención con una mayor participación de la comunidad y el equipo de salud.

En 1995 se revisa el programa estructurándolo en tres sub-programas:

1. Atención en la comunidad.

2. Atención en las instituciones.

3. Atención hospitalaria.

Su objetivo es brindar una atención integral a la población de la tercera edad, con el propósito de satisfacer sus necesidades de salud cada vez más crecientes dado el volumen de efectivos poblacionales de esta edad. ${ }^{(9)}$.

\section{Mortalidad según causas y sexo:}

Las principales causas de muerte en este grupo poblacional se corresponden con las afecciones propias de las edades avanzadas. Las tres primeras causas son: enfermedades del corazón, tumores malignos y las enfermedades cerebro vasculares, que agrupan el 62,5\% del total de las defunciones a estas edades. Se observa sobre mortalidad masculina para las causas seleccionadas, excepto para las enfermedades cerebro vasculares donde los niveles de mortalidad son ligeramente superiores en las mujeres, al igual que acontece para otros grupos de edad (Tabla 1).

Tabla 1. Principales causas de muerte en la población de 60 años y más según sexo.

\begin{tabular}{c|c|c|c|c}
\hline Causas & Masculino & Femenino & Total & Razón de tasas (M/F) \\
\hline $\begin{array}{c}\text { Enfermedades } \\
\text { del corazón }\end{array}$ & 1127,0 & 1019,6 & 1071,4 & 1,1 \\
\hline $\begin{array}{c}\text { Tumores } \\
\text { malignos }\end{array}$ & 1029,7 & 642,1 & 828,9 & 1,6 \\
\hline $\begin{array}{c}\text { Enfermedades } \\
\text { cerebro } \\
\text { vasculares }\end{array}$ & 426,5 & 448,2 & 437,7 & 1,0 \\
\hline $\begin{array}{c}\text { Influenza y } \\
\text { neumonía }\end{array}$ & 358,6 & 308,8 & 332,8 & 1,2 \\
\hline $\begin{array}{c}\text { Enf. arterias, } \\
\text { arteriolas y } \\
\text { vasos capilares }\end{array}$ & 207,5 & 183,1 & 194,8 & 1,1 \\
\hline
\end{tabular}




\section{Mortalidad por enfermedades del corazón según causas y sexo:}

En la población anciana, los mayores niveles de mortalidad por enfermedades del corazón correspondieron a la enfermedad isquémica (805,2 por 100000 hab. de la edad), siendo responsable del 75,2\% del total de las defunciones por esta causa en este grupo de edad. Para todas las causas, el riesgo de morir fue superior en los hombres (Tabla 2).

Tabla 2. Mortalidad por enfermedades del corazón en personas de 60 años y más según causas y sexo.

\begin{tabular}{c|c|c|c|c}
\hline Causas & Masculino & Femenino & Total & $\begin{array}{c}\text { Razón de tasas } \\
(\mathbf{M} / \mathbf{F})\end{array}$ \\
\hline Enfermedad isquémica & 849,4 & 764,1 & 805,2 & 1,1 \\
\hline $\begin{array}{c}\text { Otras enfermedades del } \\
\text { corazón }\end{array}$ & 189,1 & 176,8 & 182,7 & 1,1 \\
\hline $\begin{array}{c}\text { Enfermedad hipertensiva } \\
\text { Enfermedades reumáticas } \\
\text { crónicas }\end{array}$ & 84,4 & 75,1 & 79,6 & 1,1 \\
\hline
\end{tabular}

Fuente: Elaboración propia.

Tasa por 100000 habitantes de la edad y el sexo.

\section{Mortalidad por tumores malignos según localización y sexo:}

En la población de la tercera edad, los mayores niveles de mortalidad por tumores malignos para ambos sexos durante el año 2003, correspondieron al localizado en tráquea, bronquios y pulmón, seguidos por el de próstata y colon en el caso de los hombres y en el caso de las mujeres por el de colon y mama.

La sobre mortalidad masculina está presente en todas las localizaciones, excepto en el cáncer de colon, donde las cifras son superiores para el sexo femenino (95,6 defunciones por 100 000 mujeres de la edad). La mayor sobre mortalidad masculina correspondió al tumor maligno de la laringe, en el cual el riesgo de morir en los hombres es 6 veces mayor que en las mujeres (Tabla 3). 
Tabla 3. Mortalidad por tumores malignos en la población de 60 años y más según sexo y localización.

\begin{tabular}{c|c|c|c}
\hline Localización & Masculino & Femenino & $\begin{array}{c}\text { Razón de tasas } \\
(\mathbf{M} / \mathbf{F})\end{array}$ \\
\hline $\begin{array}{c}\text { Tráquea, bronquios y } \\
\text { pulmón }\end{array}$ & 284,2 & 118,2 & 2,4 \\
\hline Colon & 64,8 & 95,6 & 0,7 \\
\hline Mama & - & 84,3 & - \\
\hline Cuerpo del útero & - & 26,2 & - \\
\hline Estómago & 44,8 & 24,2 & 1,9 \\
\hline Cuello del útero & - & 17,5 & - \\
\hline Vejiga & 39,6 & 14,1 & 2,8 \\
\hline Esófago & 34,4 & 9,6 & 3,6 \\
\hline Laringe & 50,9 & 8,2 & 6,2 \\
\hline Próstata & 245,5 & - & - \\
\hline
\end{tabular}

Fuente: Elaboración propia.

Tasa por 100000 habitantes de la edad y el sexo.

\section{Otras causas de muerte seleccionadas según sexo:}

Otras entidades que alcanzan niveles de mortalidad elevados en estas edades son las infecciones respiratorias agudas (IRA) y los accidentes con tasas de 334,1 y 154,7 por 100 000 habitantes de la edad respectivamente durante el año 2003. La fractura de cadera, cuya causa accidental es la más frecuente, exhibe niveles de mortalidad de 108,6 defunciones por 100000 habitantes de la edad durante el 2003. El análisis diferencial por sexo muestra que el riesgo de morir por fractura de cadera y accidentes fue superior en las mujeres de la tercera edad. En el caso particular de los accidentes, la mayor contribución correspondió a las caídas accidentales, que en el año 2003 fueron responsables de más de la mitad de las defunciones $(63,8 \%)$ por esta causa, en esta edad y sexo. Para el resto de las causas seleccionadas se registra sobre mortalidad masculina, siendo más marcada para las lesiones auto infligidas intencionalmente en las que el riesgo de morir para los hombres es tres veces superior al de las mujeres (Tabla 4). 
Tabla 4. Otras causas de muerte en la población de 60 años y más según sexo

\begin{tabular}{c|c|c|c|c}
\hline Causas & Masculino & Femenino & Total & $\begin{array}{c}\text { Razón de } \\
\text { tasas (M/F) }\end{array}$ \\
\hline IRA & 359,7 & 310,2 & 334,1 & 1,2 \\
\hline Accidentes & 133,1 & 174,8 & 154,7 & 0,8 \\
\hline Fractura de cadera** & 71,2 & 143,4 & 108,6 & 0,5 \\
\hline $\begin{array}{c}\text { Lesiones auto } \\
\text { infligidas } \\
\text { intencionalmente }\end{array}$ & 50,9 & 16,0 & 32,8 & 3,2 \\
\hline EDA & 10,1 & 9,7 & 9,9 & 1,0 \\
\hline
\end{tabular}

Fuente: Elaboración propia.

\section{Conclusiones.}

- El envejecimiento de la población cubana es una de las implicaciones demográficas y sociales más importantes derivadas del descenso de la mortalidad y el aumento de la esperanza de vida.

- El patrón causal de mortalidad en este grupo de edad es el típico de esta etapa de la vida, y se corresponde con los reportados por países desarrollados y con estadísticas confiables. En este panorama aún persiste la influenza y neumonía con niveles de mortalidad elevados, constituyendo la cuarta causa de muerte en los hombres de 60 años y más.

- Las lesiones auto infligidas intencionalmente, alcanzan los mayores niveles de mortalidad en estas edades con franca desventaja para los hombres.

- La hipertensión arterial constituyó la enfermedad de más alta prevalencia reportada para ambos sexos, con mayores afectaciones en las mujeres. Esto la convierte en un problema de salud prioritario fundamentalmente para la cardiopatía isquémica y las enfermedades cerebros vasculares. La prevalencia elevada de esta enfermedad proviene en su gran mayoría de los grupos poblacionales de las edades precedentes, que es justamente donde es más efectiva la prevención de su aparición. Ello es válido para otros problemas de salud.

- La muerte por accidentes es también reducible en este grupo de edad por la intervención intersectorial, preparando las condiciones de movilización segura de esta población en los sitios públicos y habitacionales de sus hogares, por citar un ejemplo.

\section{Recomendaciones.}

- Incrementar el trabajo comunitario desde edades tempranas, con actividades de promoción de salud, dirigidas a cambios en estilos de vida, dirigidas fundamentalmente a la modificación de factores que constituyen riesgo para el desarrollo de enfermedades del sistema cardiovascular, el sistema osteo- 
miarticular y el sistema respiratorio.

- Realizar el Diagnóstico y Análisis de la Situación de Salud del Adulto Mayor para conocer el estado de salud de la población diana.

- Rescatar en sus funciones a los integrantes el equipo multidisciplinario de atención a la tercera edad.

- Habilitar algunos de los locales vacíos existentes en la comunidad que sus estructuras sean idóneas para la Casa del Abuelo.

- Desarrollar actividades físicas y recreativas en función de las demandas del Adulto Mayor

\section{Referencias bibliográficas.}

Albamonte, A.(1991) Ejercicios para la Tercera edad. Ed.Albatros, Argentina.

Ashford, B., Biddle, S. y Goudas, M.(1993) Participation in community sports centres: motives and predictors of enjoyment. En Journal of Sports, Health and Exercise, Staffordshire University. 11(3): 249-56, Jun.

Barboza, R. \& Alvarado, D.(1987)

Barry, HC. \& Eathorne, SW.(1994) Exercise and aging. Issues for the practitioner. Med. Clin. North. Am . 7(2): 357-76, March.

Beneficios del Ejercicio y la Actividad Física en la Tercera Edad. En la RevistaEducación,11(2):99-103.

Estado de la Población Mundial. Las nuevas generaciones. Nueva York FNUAP; 1998.

Fox. (1987) Fisiología del Deporte. Editorial Panamericana. Buenos Aires.

Guyton. (1985) Fisiología humana. Nueva Editorial Interamericana, México Fuente: http://geosalud.com/adultos_mayores/ejercicio.htm.

La Salud Pública en Cuba. Hechos y Cifras. DNE. MINSAP, Cuba 1999.

Oficina Nacional de Estadísticas. El envejecimiento de la población. Cuba y sus territorios. Cifras e indicadores para su estudio 2000. La Habana, 2001.

OPS. La Salud en las Américas. Vol I. Washington, D.C. 1998.

OPS. Las condiciones de salud en las Américas. Vol I. Washington, D.C., 1990.

Prieto Ramos O, Vega García E. Algunos sistemas de atención al anciano: programa para la atención integral al anciano. En: Temas de Gerontología. La Habana: Editorial Científico-Técnica; 1996. P.102-5. 


\section{PARA CITAR EL ARTÍCULO INDEXADO.}

González Pérez, E. R., \& Fuentes, C. M. (2018). El Adulto Mayor. Un nuevo reto en El Milenio. Anatomía Digital, 1(2), 15-28. https://doi.org/10.33262/anatomiadigital.v1i2.1043

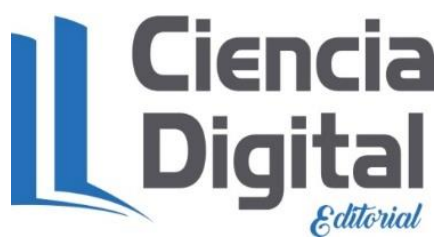

El artículo que se publica es de exclusiva responsabilidad de los autores y no necesariamente reflejan el pensamiento de la Revista Anatomía Digital.

El artículo queda en propiedad de la revista y, por tanto, su publicación parcial y/o total en otro medio tiene que ser autorizado por el director de la Revista Anatomía Digital.
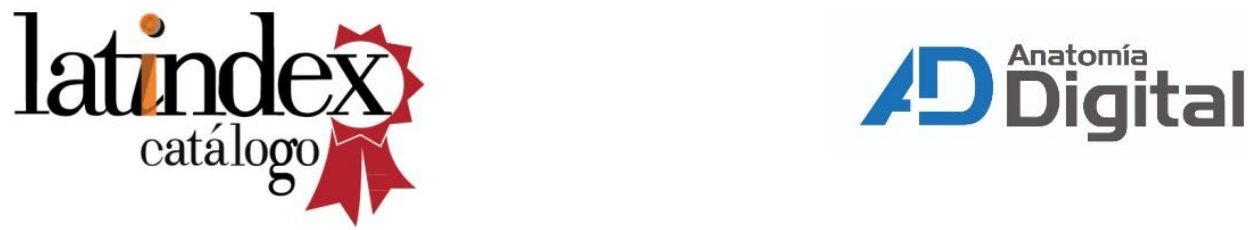